\title{
Congenital bleeding disorders and dental surgery
}

\section{Abstracted from}

\section{Coppola A, Windyga J, Tufano A, Yeung C, Di Minno MND}

Treatment for preventing bleeding in people with haemophilia or other congenital bleeding disorders undergoing surgery. Cochrane Database Syst Rev 2015; 2: Art. No. CD009961. DOI: 10.1002/14651858.CD009961.pub2.

Address for correspondence: Antonio Coppola, Regional Reference Centre for Coagulation Disorders,

Dept of Clinical Medicine and Surgery, Federico II University, Naples, Italy. E-mail: antocopp@unina.it.

\section{Question: What is the most effective and safe treatment to prevent bleeding in people with congenital bleeding disorders undergoing surgery?}

Data sources Cochrane Cystic Fibrosis and Genetic Disorders Group's Coagulopathies Trials Register. This register is updated regularly from the Cochrane Library weekly searches of Medline and prospective hand-searching of the journal, Haemophilia. ClinicalTrials.gov was searched for possible ongoing trials.

Study selection Two reviewers independently selected studies randomised and quasi-randomised studies were considered.

Data extraction and synthesis Two reviewers independently extracted data and assessed risk of bias using the Cochrane risk of bias tool. Meta-analysis was conducted.

Results Three RCTs and one quasi-RCT involving a total of 112 patients were included. Two of the trials (one RCT, one quasi$\mathrm{RCT}$ ) involving a total of 59 people were conducted in patients undergoing dental extractions. They compared the use of a different type (tranexamic acid or epsilon-aminocaproic acid) and regimen of antifibrinolytic agents as haemostatic support to the initial replacement treatment. They found a reduction of blood loss and requirement of post-operative replacement treatment in people receiving antifibrinolytic agents compared with placebo. The need for re-intervention was not reported by either trial.

Conclusions There is insufficient evidence from randomised controlled trials to assess the most effective and safe haemostatic treatment to prevent bleeding in people with haemophilia or other congenital bleeding disorders undergoing surgical procedures. Ideally large, adequately powered and well-designed randomised controlled trials would be needed, in particular to address the cost-effectiveness of such demanding treatments in the light of the increasing present economic constraints, and to explore the new challenge of ageing patients with haemophilia or other congenital bleeding disorders. However, performing such trials is always a complex task in this setting and presently does not appear to be a clinical and research

This paper is based on a Cochrane Review published in the Cochrane Library 2015, issue 2 (see www.thecochranelibrary.com for information). Cochrane Reviews are regularly updated as new evidence emerges and in response to feedback, and the Cochrane Library should be consulted for the most recent version of the review. priority. Indeed, major and minor surgeries are effectively and safely performed in these individuals in clinical practice, with the numerous national and international recommendations and guidelines providing regimens for treatment in this setting mainly based on data from observational, uncontrolled studies.

\section{Commentary}

For many dentists, a patient with haemophilia is someone who requires a referral to a specialty centre for all aspects of oral care. For those delivering care to this patient population it is very important to rely on the best quality evidence, especially during invasive procedures.

Haemophilia is a genetic bleeding disorder caused by a mutation in different genes. It is the most common inherited factor deficiency. While in haemophilia A the mutation affects coagulation factor VIII, in haemophilia B the coagulation factor IX is affected. Haemophilia A is four times more common, affecting one in 5,000 to 10,000 males worldwide. For the most part these are inherited in an X-linked recessive manner. Severity classification depends on the factor levels, which could be as low as $<1 \%$ of normal values in severe cases. Sixty percent of the cases are classified as severe. Severity is also related to spontaneous and excessive bleeding after trauma and injury.

Patients with haemophilia are usually treated with infusions of recombinant factor products or plasma-derived factor products, depending on severity and availability.

Antibodies, also known as inhibitors, can develop over time, especially in severe cases of type A haemophilia. Treating patients with an inhibitor is especially complex and challenging. Other medications, like DDAVP (desmopressin), aminocaproic acid and tranexamic acid are also used for treatment around surgical procedures.

Post-operative bleeding after surgical procedures is not uncommon but certainly an unwanted complication that should be prevented if possible in any dental surgical procedure. However, in patients with haemophilia or other congenital bleeding disorders it is extremely important to plan and monitor the blood loss in advance.

The question selected by the Cochrane Cystic Fibrosis and Genetic Disorders group intended to find randomised or quasirandomised controlled trials assessing the outcome of mortality and blood loss as the main primary outcome in minor and major surgical procedures. Haemostasis effectiveness, use of alternative haemostatic treatment, and achievement of haemostatic levels were 
considered as secondary outcomes. Unsurprisingly, the authors were not able to find randomised controlled trials assessing mortality as the main outcome.

They included randomised and quasi-randomised studies, which are appropriate for assessing effectiveness, but controversial for learning about complications and negative outcomes. After a comprehensive search including Medline, trial registries, handsearching and unpublished work from five major conferences, two of the authors independently assessed inclusion, and a protocol in case of disagreement was followed. Four trials were eligible, all of them including adults and children with haemophilia with different degrees of severity. Each author evaluated the risk of bias criteria following the Cochrane Handbook.

The authors identified two categories: one with and one without the presence of inhibitors. Interestingly, the two trials without inhibitors $(n=59)$ are from the early seventies, and were conducted on patients undergoing dental extractions comparing the use of two antifibrinolytic agents: tranexamic acid or aminocaproic acid to placebo. While the antifibrinolytic agents are still in use, the factor concentrates used at that time are now seldom recommended. Both trials assessed variation of haemoglobin levels, though in different manners. They showed a statistical advantage for using both agents over placebo, which was more modest in the case of aminocaproic acid.

The two trials on patients with inhibitors $(\mathrm{n}=53)$ undergoing major or minor elective surgery compared different regimens of recombinant factor VIIa. The individual studies may not provide enough power to show differences, and the haemostatic efficacy was assessed rather subjectively.

The methodology of the review was acceptable, however for the selected inclusion criteria only a few trials were included. As the authors concluded, they are published articles that provide help to practitioners. ${ }^{1,2.3 .4}$

Congenital bleeding disorders are not as infrequent as is generally considered.
The deficiency predisposes to recurrent joint, muscle and mucosal bleeds and not infrequently, intracranial and intraperitoneal haemorrages can occur. Bleeding frequency and severity is proportional to the deficiency. ${ }^{5,6}$ Medical and dental management may differ depending on the bleeding disorder and the intervention expected.

Practitioners should be prepared to manage patients with congenital bleeding disorders and have communication with the patient's medical providers.

It is important to note that the landscape of treatment could soon change dramatically because of advancements in gene therapy. In the meantime, the inconclusive nature of this review and the limitations of the included studies highlight the importance of being familiar with other resources, like the World Federation of Hemophilia ${ }^{7}$ or the "Guidance on the dental management of patients with haemophilia and congenital bleeding disorders. ${ }^{1}$ Future research will benefit from the examination of patientcentred outcomes like preferences and pain levels, and the cost of treatment.

Silvia Spivakovsky, Analia Veitz-Keenan, Marie Congiusta and Yael Spivakovsky NYU College of Dentistry, New York, USA

1. Anderson JA, Brewer A, Creagh D, et al. Guidance on the dental management of patients with haemophilia and congenital bleeding disorders. Br Dent J 2013; 215: 497-504.

2. Brewer AK, Roebuck EM, Donachie M, et al. The dental management of adult patients with haemophilia and other congenital bleeding disorders. Haemophilia 2003; 9: 673-677

3. Martlew VJ. Peri-operative management of patients with coagulation disorders. Br J Anaesth 2000; 85: 446-455.

4. http://www1.wfh.org/publication/files/pdf-1472.pdf. Guidelines for the management of hemophilia. 2nd edition. [Accessed 26 August 2015].

5. Peyvandi F, Mannucci PM. Rare coagulation disorders. Thromb Haemost 1999; 82: 1207-1214.

6. Prevention and control of haemophilia: memorandum from a joint WHO/WFH meeting. Bulletin of the World Health Organization 1991; LXIX: 17-26

7. http://www.wfh.org

Evidence-Based Dentistry (2015) 16, 90-91. doi:10.1038/sj.ebd.6401121 